\title{
ASC amino-acid transporter 2 (ASCT2) as a novel prognostic marker in non-small cell lung cancer
}

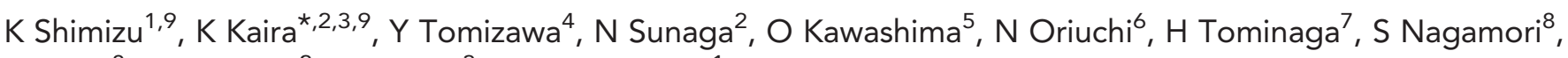
Y Kanai ${ }^{8}$, M Yamada ${ }^{2}$, T Oyama ${ }^{3}$ and I Takeyoshi ${ }^{1}$

${ }^{1}$ Department of Thoracic and Visceral Surgery, Gunma University Graduate School of Medicine, Showa-machi, Maebashi, Gunma 371-8511, Japan; 'Department of Medicine and Molecular Science, Gunma University Graduate School of Medicine, Showa-machi, Maebashi, Gunma, Japan; ${ }^{3}$ Department of Diagnostic Pathology, Gunma University Graduate School of Medicine, Showa-machi, Maebashi, Gunma, Japan; ${ }^{4}$ Department of Internal Medicine, NHO Nishi-Gunma Hospital, 2854 Kanai Shibukawa, Gunma, Japan; ${ }^{5}$ Department of Surgery, NHO Nishi-Gunma Hospital, 2854 Kanai Shibukawa, Gunma, Japan; ${ }^{6}$ Department of Diagnostic Radiology and Nuclear Medicine, Gunma University Graduate School of Medicine, Showa-machi, Maebashi, Gunma, Japan; 'Department of Molecular Imaging, Gunma University Graduate School of Medicine, Showa-machi, Maebashi, Gunma, Japan and ${ }^{8}$ Division of Bio-system Pharmacology, Department of Pharmacology, Graduate School of Medicine, Osaka University, Osaka, Japan
\end{abstract}

Background: ASC amino-acid transporter 2 (ASCT2) is a major glutamine transporter that has an essential role in tumour growth and progression. Although ASCT2 is highly expressed in various cancer cells, the clinicopathological significance of its expression in non-small cell lung cancer (NSCLC) remains unclear.

Methods: One hundred and four patients with surgically resected NSCLC were evaluated as one institutional cohort. Tumour sections were stained by immunohistochemistry (IHC) for ASCT2, Ki-67, phospho-mTOR (mammalian target of rapamycin), and CD34 to assess the microvessel density. Two hundred and four patients with NSCLC were also validated by IHC from an independent cohort.

Results: ASC amino-acid transporter 2 was expressed in $66 \%$ of patients, and was closely correlated with disease stage, lymphatic permeation, vascular invasion, CD98, cell proliferation, angiogenesis, and mTOR phosphorylation, particularly in patients with adenocarcinoma (AC). Moreover, two independent cohorts confirmed that ASCT2 was an independent marker for poor outcome in $\mathrm{AC}$ patients.

Conclusions: ASC amino-acid transporter 2 expression has a crucial role in the metastasis of pulmonary AC, and is a potential molecular marker for predicting poor prognosis after surgery.

Lung cancer is the leading cause of cancer deaths worldwide. Therefore, assessing the potential of established biomarkers for predicting the outcome and the response to specific therapies is important to improve the prognosis of patients with non-small cell lung cancer (NSCLC). Tumour staging and performance status are currently the most powerful prognostic predictors in patients with NSCLC (Brundage et al, 2002). Recent large-scale studies demonstrated that sex, smoking history, and histology could affect

\footnotetext{
*Correspondence: Dr K Kaira; E-mail: kkaira1970@yahoo.co.jp

${ }^{9}$ These authors contributed equally to this work.
}

Received 14 December 2013; revised 19 January 2014; accepted 23 January 2014; published online 6 March 2014 (C) 2014 Cancer Research UK. All rights reserved 0007 - 0920/14 
the prognosis after treatment in patients with NSCLC, especially in adenocarcinoma (AC) (Kawaguchi et al, 2010; Nakamura et al, 2011; Kogure et al, 2013). Performance status and disease staging are generally known factors associated with prognosis after treatment.

ASC amino-acid transporter 2 (ASCT2) is a $\mathrm{Na}^{+}$-dependent transporter responsible for the transport of neutral amino acids, including glutamine, leucine, and isoleucine (Kekuda et al, 1996). It is the major glutamine transporter in human hepatoma cells (Fuchs et al, 2007), and has a role in tumour growth and the proliferation of cancer cells (Fuchs and Bode, 2006). It is highly expressed in various malignancies, including hepatocellular carcinoma and colorectal or prostate cancer, and its expression is closely associated with tumour aggressiveness and prognosis in colorectal or prostate cancer (Whitte et al, 2002; Li et al, 2003; Fuchs et al, 2007). It provides cancer cells with essential amino acids for protein synthesis, and it coordinates tumour cell growth through the activation of mammalian target of rapamycin (mTOR) (Fuchs et al, 2007). Glutamine promotes cancer cell proliferation and has a high affinity for ASCT2 (Fuchs and Bode, 2006; Fuchs et al, 2007). Amino-acid transporters are essential for the growth and survival of tumour cells, and L-type amino-acid transporter 1 (LAT1) also has a crucial role in the development and proliferation of transformed cells (Kanai et al, 1998; Yanagida et al, 2001; Kaira et al, 2008, 2012). It is an L-type amino-acid transporter that transports large neutral amino acids, such as leucine, isoleucine, valine, phenylalanine, tyrosine, tryptophan, methionine, and histidine (Kanai et al, 1998; Yanagida et al, 2001). It requires a covalent association with the heavy chain of 4F2 cell-surface antigen (CD98) for its functional expression and localisation in the plasma membrane (Kanai et al, 1998; Yanagida et al, 2001). Recent studies have focussed on ASCT2 and LAT1, which are highly expressed in cancer cells (Fuchs and Bode, 2006). The overexpression of LAT1 may be a significant predictor of poor prognosis, and it is closely linked to the aggressiveness and metastasis of various human neoplasms (Nawashiro et al, 2006; Nakanishi et al, 2007; Kaira et al, 2008, 2012; Sakata et al, 2009; Ichinoe et al, 2011; Furuya et al; 2012). Although the clinical importance of LAT1 expression in cancer cells is understood, the clinicopathological significance of ASCT2 expression in human neoplasms remains unclear. We therefore conducted a clinicopathological study to investigate the expression of ASCT2 in tissue specimens of resected NSCLC. The aim of our study was to clarify whether the expression of ASCT2 was closely associated with the outcome after treatment and to explore the relationship between ASCT2 and clinical characteristics. In addition, the correlation between ASCT2 expression and CD98, the Ki-67 labelling index (LI), microvessel density (MVD) (determined by CD34), and the phosphorylation of mTOR (p-mTOR) was assessed.

\section{MATERIALS AND METHODS}

Patients. We analysed 111 consecutive patients with NSCLC who underwent resection either by lobectomy or pneumonectomy with mediastinal lymph-node dissection at Nishigunma National Hospital (NGH, Shibukawa, Japan) between July 2007 and January 2010. Of these patients, 7 were excluded from further analysis because tissue specimens were not available; thus, 104 patients were enrolled in the study. Postoperative adjuvant chemotherapy with platinum-based regimens, S-1 (Taiho Pharmaceutical Co., Ltd, Tokyo, Japan) and oral administration of tegafur (a fluorouracil derivative drug) were administered to 19, 1, and 12 patients, respectively. No chemotherapy or radiotherapy before surgery was performed on any patient. The study protocol was approved by the institutional review board. The tumour specimens were histologically classified according to World Health Organisation criteria. The stages of pathological tumour-node-metastasis were established using the International System for Staging Lung Cancer adopted by the American Joint Committee on Cancer and the Union Internationale Centre le Cancer (Mountain, 1997). The day of surgery was considered to be the first day after surgery. The follow-up duration ranged from 139 to 2118 days (median, 1362 days).

For validation, we analysed an independent series of patients with NSCLC who underwent complete resection of the primary lung tumour with mediastinal lymph-node dissection at Gunma University Hospital (GUH, Maebashi, Japan) between June 2003 and June 2008. The median follow-up period was 1932 days (range, 160-3765 days).

Immunohistochemical staining. The protocol used for immunohistochemistry (IHC) is described elsewhere (Kaira et al, 2008, 2012). An oligopeptide (RDSKGLAAAEPTAN), corresponding to amino acids $7-20$ of ASCT2 (1:300 dilution), was used to synthesise rabbit polyclonal antibodies, as described previously (Altman et al, 1984). The N-terminal cysteine residue was used for conjugation to keyhole limpet haemocyanin. The antiserum was affinity purified as described previously (Chairoungdua et al, 2001), and the specificity was confirmed (Supplementary Procedures). Briefly, HEK293T cells were transfected with a plasmid encoding ASCT2 or empty vector control. Crude membrane fractions were isolated, separated by SDS-PAGE, and analysed by western blotting as described by Khunweeraphong et al (2012). Immunohistochemistry was performed on paraffin sections using the polymer peroxidase method (Histofine Simple Stain MAX PO (MULTI) kit; Nichirei Corp., Tokyo, Japan). Briefly, deparaffinised and rehydrated sections were treated with $0.3 \%$ hydrogen peroxide $\left(\mathrm{H}_{2} \mathrm{O}_{2}\right)$ in methanol for $30 \mathrm{~min}$ to block endogenous peroxidase activity. To expose the antigens, sections were autoclaved in ethylenediaminetetraacetic acid ( $\mathrm{pH}$ 8.0) for
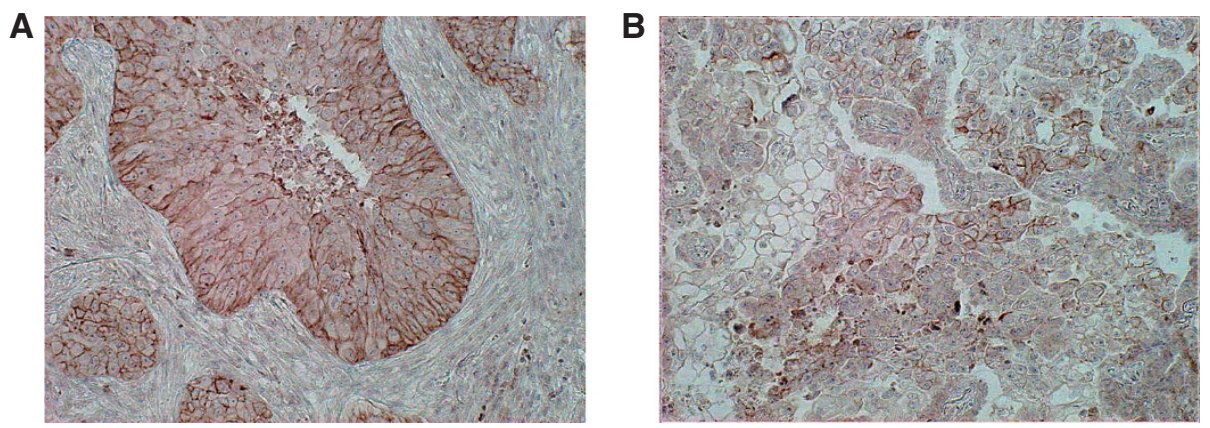

Figure 1. Immunohistochemical staining of tumour tissue from a 68-year-old male with a pulmonary SQC (A) and a 70-year-old female with a pulmonary AC (B). ASCT2 exhibited a membranous immunostaining pattern (A, score of 4; $B$, score of 3 ). 
$5 \mathrm{~min}$ and cooled for $30 \mathrm{~min}$. After rinsing in phosphate-buffered saline, the sections were incubated with affinity-purified antiASCT2 antibodies $(1: 300)$ overnight followed by immunohistochemical staining with a Histofine Simple Stain MAX PO (MULTI) kit (Nichirei Corp.). The peroxidase reaction was carried out using $0.02 \% 3,3^{\prime}$-diaminobenzidine tetrahydrochloride and $0.01 \% \mathrm{H}_{2} \mathrm{O}_{2}$ in $0.05 \mathrm{M}$ Tris- $\mathrm{HCl}$ ( $\mathrm{pH} 7.4$ ). Negative control tissue sections were stained as described above, except that the primary antibody was omitted.

Anti-CD98 is an affinity-purified rabbit polyclonal antibody ( $1: 100$ dilution; Santa Cruz Biotechnology Inc., Santa Cruz, CA, USA) raised against a C-terminal peptide of human CD98. ASC amino-acid transporter 2 and CD98 staining was considered as positive only if distinct membrane staining was detected. The ASCT2 and CD98 expression scores were assessed by the extent of staining as follows: $1, \leqslant 10 \%$ of the tumour area stained; 2 , $11-25 \% ; 3,26-50 \%$; and $4, \geqslant 51 \%$ stained. Those tumours with a score of $>2$ were considered to have a high level of expression.

Mouse monoclonal antibodies against CD34 (1:800 dilution; Nichirei Corp.) and Ki-67 (1:40; Dako, Glostrup, Denmark), and a rabbit monoclonal antibody against $\mathrm{p}-\mathrm{mTOR}(1: 80$; Cell Signaling Technology, Danvers, MA, USA) were also used. The number of CD34-positive vessels was counted in four randomly selected regions in a $\times 400$ field $\left(0.26 \mathrm{~mm}^{2}\right.$ field area). The MVD was defined as the mean number of microvessels per $0.26 \mathrm{~mm}^{2}$ field area, and tumours in which the number of stained tumour cells was greater than the median were defined as high expressors. For $\mathrm{Ki}-67$, epithelial cells with nuclear staining of any intensity were considered to be positive. Approximately 1000 nuclei were counted on each slide, and the proliferative activity was assessed as the percentage of Ki-67-stained nuclei (Ki-67 LI) in each sample. The median Ki-67 LI was evaluated, and tumours with an LI greater than the median were considered to be positive. For p-mTOR, a semiquantitative scoring method was used: $1,<10 \% ; 2,10-25 \% ; 3$, $25-50 \%$; and $4, \geqslant 51 \%$ of positive cells. Those tumours with a staining score of $>3$ were considered to be strongly stained (Kaira et al, 2008, 2012). All sections were independently assessed using light microscopy in a blinded manner by at least two of the authors.

Statistical analysis. $P$-values $<0.05$ were used to indicate a statistically significant difference. Fisher's exact test was used to examine the association between two categorical variables. The correlation between different variables was analysed using the nonparametric Spearman's rank test. ASC amino-acid transporter 2 expression score was correlated with other immunohistochemical measurements and clinical variables. Since the sample size of NGH was not enough to do multivariate analysis using many prognostic variables; sex, smoking, stage, and histology which are known factors as described above were selected for the analysis. We added ASCT2 to these variables, and multivariate analysis was performed. In GUH cohort, we did multivariate analysis using the same prognostic variables.

Elderly patients were defined as more than 65 years old, and an ever smoker was defined as someone who had smoked at least 100 cigarettes in his lifetime. Disease staging was divided into two groups; stage I or II (early disease) and stage III or IV (advanced disease). The Kaplan-Meier method was used to estimate survival as a function of time, and survival differences were analysed by the log-rank test. Overall survival (OS) was determined as the time from tumour resection to death from any cause. Progression-free survival (PFS) was defined as the time between tumour resection and the first disease progression or death. Multivariate analyses were performed using a stepwise Cox proportional hazards model to identify independent prognostic factors. Statistical analyses were performed using JMP 8 for Windows (SAS Institute Inc., Cary, NC, USA).
Table 1. Demographics and clinical characteristics of the patients

\begin{tabular}{l|c|c|c|}
\hline Variables & $\begin{array}{c}\text { NGH cohort } \\
(\boldsymbol{n}=104)\end{array}$ & $\begin{array}{c}\text { GUH cohort } \\
(\boldsymbol{n}=\mathbf{2 0 4})\end{array}$ & $P$-value \\
\hline Age & 31 & 67 & 0.607 \\
\hline$\leqslant 65$ years & 73 & 137 & \\
$>65$ years & &
\end{tabular}

\begin{tabular}{|l|r|r|l|}
\hline \multicolumn{4}{|l|}{ Sex } \\
\hline Male & 64 & 119 & 0.624 \\
Female & 40 & 85 & \\
\hline
\end{tabular}

\section{Smoking}

\begin{tabular}{|l|r|r|r}
\hline Yes & 66 & 126 & 0.805 \\
No & 38 & 78 & \\
\hline
\end{tabular}

p-Stage

\begin{tabular}{|l|r|r|r}
\hline I or II & 80 & 159 & 0.658 \\
III or IV & 24 & 41 & \\
\hline
\end{tabular}

III or IV

T factor

\begin{tabular}{|l|r|r|r|}
\hline T1-2 & 93 & 177 & 0.585 \\
T3-4 & 11 & 27 & \\
\hline
\end{tabular}

$\mathrm{N}$ factor

\begin{tabular}{|l|r|r|r|}
\hline N0 & 71 & 143 & 0.795 \\
N1-2 & 33 & 61 & \\
\hline
\end{tabular}

\section{Histology}

\begin{tabular}{|l|r|r|r}
\hline AC & 66 & 142 & 0.304 \\
\hline
\end{tabular}

Non-AC

Lymphatic permeation

\begin{tabular}{|l|r|r|r|}
\hline Positive & 59 & 87 & $\mathbf{0 . 0 2 2}$ \\
Negative & 45 & 117 & \\
\hline
\end{tabular}

Negative 45

Vascular invasion

\begin{tabular}{l|r}
\hline Positive & 57
\end{tabular}

Negative 57

47

0.001

\section{ASCT2}

\begin{tabular}{|l|l|l|l|}
\hline High & 66 & 101 & 0.022 \\
Low & 38 & 103 & \\
\hline
\end{tabular}

\section{CD98}

\begin{tabular}{|l|r|r|r|}
\hline High & 57 & 68 & $<0.001$ \\
Low & 47 & 136 & \\
\hline
\end{tabular}

\section{$\mathrm{Ki}-67$}

\begin{tabular}{|l|r|r|r|}
\hline High & 46 & 90 & $>0.999$ \\
Low & 58 & 114 & \\
\hline
\end{tabular}

\section{CD34}

\begin{tabular}{|l|r|r|r|}
\hline High & 47 & 69 & 0.062 \\
Low & 57 & 135 & \\
\hline
\end{tabular}

\section{p-mTOR}

\begin{tabular}{|l|r|r|r|}
\hline High & 41 & 56 & 0.038 \\
Low & 63 & 148 & \\
\hline
\end{tabular}

Abbreviations: $\mathrm{AC}=$ adenocarcinoma; $\mathrm{ASCT} 2=\mathrm{ASC}$ amino-acid transporter 2; $\mathrm{GUH}=\mathrm{Gunma}$ University Hospital; $\mathrm{NGH}=$ Nishi-Gunma Hospital; non-AC = non-adenocarcinoma; $\mathrm{p}-\mathrm{mTOR}=$ phosphorylation of mammalian target of rapamycin.

Bold entries show statistically significant difference. 
Table 2. Patient's demographics according to ASCT2 expression in NGH cohort

\begin{tabular}{|c|c|c|c|c|c|c|c|c|c|c|}
\hline & & \multicolumn{3}{|c|}{ All $(n=104)$} & \multicolumn{3}{|c|}{$A C(n=66)$} & \multicolumn{3}{|c|}{ Non-AC $(n=38)$} \\
\hline Variable & $\begin{array}{c}\text { Total } \\
(n=104)(\%)\end{array}$ & $\begin{array}{c}\text { High } \\
(n=66)\end{array}$ & $\begin{array}{c}\text { Low } \\
(n=38)\end{array}$ & $P$-value & $\begin{array}{c}\text { High } \\
(n=36)\end{array}$ & $\begin{array}{c}\text { Low } \\
(n=30)\end{array}$ & $P$-value & $\begin{array}{c}\text { High } \\
(n=30)\end{array}$ & $\begin{array}{l}\text { Low } \\
(n=8)\end{array}$ & $P$-value \\
\hline \multicolumn{11}{|l|}{ Age } \\
\hline $\begin{array}{l}\leqslant 65 \text { years } \\
>65 \text { years }\end{array}$ & $\begin{array}{l}31 \\
73\end{array}$ & $\begin{array}{l}23 \\
43\end{array}$ & $\begin{array}{r}8 \\
30\end{array}$ & 0.182 & $\begin{array}{l}18 \\
18\end{array}$ & $\begin{array}{r}7 \\
23\end{array}$ & 0.041 & $\begin{array}{r}5 \\
25\end{array}$ & $\begin{array}{l}1 \\
7\end{array}$ & $>0.999$ \\
\hline
\end{tabular}

\section{Sex}

\begin{tabular}{|l|l|l|l|l|l|l|l|r|r|r|r|r|} 
Male & 64 & 46 & 18 & 0.035 & 19 & 12 & 0.332 & 27 & 6 & 0.279 \\
Female & 40 & 20 & 20 & & 17 & 18 & & 3 & 2 & \\
\hline
\end{tabular}

\section{Smoking}

\begin{tabular}{|l|l|l|l|l|l|l|l|r|r|r|r|r|r|}
\hline Yes & 66 & 45 & 21 & 0.209 & 17 & 14 & $>0.999$ & 28 & 7 \\
No & 38 & 21 & 17 & & 19 & 16 & & 2.518 & 1 & 2 \\
\hline
\end{tabular}

\section{p-Stage}

\begin{tabular}{|l|l|l|r|r|r|r|r|r|r|r|r|r|r|r|}
\hline III or IV & 80 & 44 & 36 & 0.001 & 20 & 30 & $<0.001$ & 24 & 6 & $>0.999$ \\
\hline
\end{tabular}

\section{T factor}

\begin{tabular}{|c|c|c|c|c|c|c|c|c|c|c|}
\hline $\begin{array}{l}\text { T1-2 } \\
\text { T3-4 }\end{array}$ & $\begin{array}{l}93 \\
11\end{array}$ & $\begin{array}{l}55 \\
11\end{array}$ & $\begin{array}{r}38 \\
0\end{array}$ & 0.006 & $\begin{array}{r}27 \\
9\end{array}$ & $\begin{array}{r}30 \\
0\end{array}$ & 0.003 & $\begin{array}{r}28 \\
2\end{array}$ & $\begin{array}{l}8 \\
0\end{array}$ & $>0.999$ \\
\hline \multicolumn{11}{|c|}{$\mathbf{N}$ factor } \\
\hline $\begin{array}{l}\text { N0 } \\
\text { N1-2 }\end{array}$ & $\begin{array}{l}71 \\
33\end{array}$ & $\begin{array}{l}40 \\
26\end{array}$ & $\begin{array}{r}31 \\
7\end{array}$ & 0.030 & $\begin{array}{l}18 \\
18\end{array}$ & $\begin{array}{r}25 \\
5\end{array}$ & 0.009 & $\begin{array}{r}22 \\
8\end{array}$ & $\begin{array}{l}6 \\
2\end{array}$ & $>0.999$ \\
\hline
\end{tabular}

\section{Histology}

\begin{tabular}{l|l|l|r|r}
\hline AC & 66 & 36 & 30 & 0.019 \\
Non-AC & 38 & 30 & 8 & \\
\hline
\end{tabular}

\section{Lymphatic permeation}

\begin{tabular}{|l|r|r|r|r|r|r|r|r|r|r|r|}
\hline Positive & 59 & 44 & 15 & 0.008 & 27 & 12 & 0.006 & 17 & 3 & 0.438 \\
Negative & 45 & 22 & 23 & & 9 & 18 & & 13 & 5 & \\
\hline
\end{tabular}

\section{Vascular invasion}

\begin{tabular}{|c|c|c|c|c|c|c|c|c|c|c|}
\hline Positive & 57 & 44 & 13 & 0.002 & $\begin{array}{l}17 \\
19\end{array}$ & $\begin{array}{r}8 \\
22\end{array}$ & 0.126 & $\begin{array}{r}27 \\
3\end{array}$ & $\begin{array}{l}5 \\
3\end{array}$ & 0.094 \\
\hline \multicolumn{11}{|l|}{ CD98 } \\
\hline $\begin{array}{l}\text { High } \\
\text { Low }\end{array}$ & $\begin{array}{l}57 \\
47\end{array}$ & $\begin{array}{l}46 \\
20\end{array}$ & $\begin{array}{l}11 \\
27\end{array}$ & $<0.001$ & $\begin{array}{l}20 \\
16\end{array}$ & $\begin{array}{r}4 \\
26\end{array}$ & $<0.001$ & $\begin{array}{r}26 \\
4\end{array}$ & $\begin{array}{l}7 \\
1\end{array}$ & $>0.999$ \\
\hline
\end{tabular}

\section{Ki-67}

\begin{tabular}{l|l|l|l|l|l|l|l|r|r|r|r|r|r|r|} 
High & 46 & 42 & 10 & $<0.001$ & 13 & 5 & 0.099 & 29 & 5 \\
Low & 58 & 24 & 28 & & 23 & 25 & & 1 & 3 & \\
\hline
\end{tabular}

\section{CD34}

\begin{tabular}{l|l|l|l|l|l|l|l|l|l|l|l|} 
High & 47 & 38 & 9 & 0.001 & 22 & 5 & $<0.001$ & 16 & 4 & $>0.999$ \\
Low & 57 & 28 & 29 & & 14 & 25 & & 14 & 4 & \\
\hline
\end{tabular}

\section{p-mTOR}

\begin{tabular}{|l|l|l|l|l|l|l|l|l|l|r|r|}
\hline High & 41 & 30 & 11 & 0.144 & 26 & 11 & 0.006 & 4 & 0 & 0.559 \\
Low & 63 & 36 & 27 & & 10 & 19 & & 26 & 8 & \\
\hline
\end{tabular}

Abbreviations: $\mathrm{AC}=$ adenocarcinoma; $\mathrm{ASCT} 2=\mathrm{ASC}$ amino-acid transporter 2; $\mathrm{NGH}=$ Nishi-Gunma Hospital; non- $\mathrm{AC}=$ non-adenocarcinoma; $\mathrm{p}-\mathrm{mTOR}=$ phosphorylation of mammalian target of rapamycin; $\mathrm{p}$-stage $=$ pathological stage .

Bold entries show statistically significant difference. 


\section{RESULTS}

Immunohistochemical analysis and clinicopathological features. One hundred and four primary lung cancer lesions were analysed by IHC. Figure 1 shows representative staining for ASCT2.
Expression of ASCT2 was detected in carcinoma cells in tumour tissues, and it was localised predominantly on the plasma membrane. All positive cells showed strong membrane staining. High levels of ASCT2 and CD98 expression were observed in 63\% (66 out of 104) and 55\% (55 out of 104) of the tumours, respectively. When staining was correlated with histological type, a

Table 3. Univariate and multivariate analysis of overall survival and progression-free survival in NGH cohort

\begin{tabular}{|c|c|c|c|c|c|c|}
\hline \multirow[b]{3}{*}{ Variables } & \multicolumn{3}{|c|}{ Overall survival } & \multicolumn{3}{|c|}{ Progression-free survival } \\
\hline & \multicolumn{2}{|c|}{ Univariate analysis } & \multirow{2}{*}{$\begin{array}{c}\text { Multivariate } \\
\text { analysis }\end{array}$} & \multicolumn{2}{|c|}{ Univariate analysis } & \multirow{2}{*}{$\begin{array}{c}\text { Univariate analysis } \\
\text { HR (95\% CI) } \\
P \text {-value }\end{array}$} \\
\hline & $\begin{array}{l}\text { 5-Year } \\
\text { survival } \\
\text { rate (\%) }\end{array}$ & $\begin{array}{c}\text { HR } \\
95 \% \mathrm{Cl} \\
P \text {-value }\end{array}$ & & $\begin{array}{c}\text { 5-year survival } \\
\text { rate }(\%)\end{array}$ & $\begin{array}{c}\text { HR } \\
P \text {-value }\end{array}$ & \\
\hline Age & & 0.959 & & & 1.640 & \\
\hline $\begin{array}{l}\leqslant 65 \text { years } \\
>65 \text { years }\end{array}$ & $\begin{array}{l}46 \\
54\end{array}$ & $\begin{array}{c}0.513-1.796 \\
0.897\end{array}$ & & $\begin{array}{l}33 \\
55\end{array}$ & $\begin{array}{c}0.884-3.042 \\
0.116\end{array}$ & \\
\hline Sex & & 2.322 & 1.326 & & 1.978 & \\
\hline $\begin{array}{l}\text { Male } \\
\text { Female }\end{array}$ & $\begin{array}{l}44 \\
66\end{array}$ & $\begin{array}{c}1.284-4.200 \\
0.005\end{array}$ & $\begin{array}{c}0.819-2.174 \\
0.255\end{array}$ & $\begin{array}{l}40 \\
61\end{array}$ & $\begin{array}{c}1.130-3.463 \\
0.017\end{array}$ & $\begin{array}{c}2.074(0.857-5.031) \\
0.106\end{array}$ \\
\hline Smoking & & 1.821 & 0.898 & & 1.314 & \\
\hline $\begin{array}{l}\text { Yes } \\
\text { No }\end{array}$ & $\begin{array}{l}47 \\
61\end{array}$ & $\begin{array}{c}1.001-3.299 \\
0.048\end{array}$ & $\begin{array}{c}0.316-2.359 \\
0.834\end{array}$ & $\begin{array}{l}46 \\
53\end{array}$ & $\begin{array}{c}0.746-2.313 \\
0.344\end{array}$ & $\begin{array}{c}1.598(0.646-3.713) \\
0.302\end{array}$ \\
\hline $\mathrm{p}$-Stage & & 6.605 & 2.677 & & 9.022 & \\
\hline $\begin{array}{l}\text { I or II } \\
\text { III or IV }\end{array}$ & $\begin{array}{l}62 \\
10\end{array}$ & $\begin{array}{c}2.929-14.89 \\
<0.001\end{array}$ & $\begin{array}{c}1.394-5.079 \\
0.004\end{array}$ & $\begin{array}{l}59 \\
14\end{array}$ & $\begin{array}{c}4.027-20.21 \\
<0.001\end{array}$ & $\begin{array}{c}2.935(1.574-5.420) \\
<\mathbf{0 . 0 0 1}\end{array}$ \\
\hline Histology & & 1.985 & 1.274 & & 1.388 & \\
\hline $\begin{array}{l}\text { AC } \\
\text { Non-AC }\end{array}$ & $\begin{array}{l}57 \\
42\end{array}$ & $\begin{array}{c}1.047-3.763 \\
0.035\end{array}$ & $\begin{array}{c}0.648-2.533 \\
0.482\end{array}$ & $\begin{array}{l}51 \\
44\end{array}$ & $\begin{array}{c}0.768-2.507 \\
0.277\end{array}$ & $\begin{array}{c}0.965(0.509-1.835) \\
0.915\end{array}$ \\
\hline Lymphatic permeation & & 2.517 & & & 2.543 & \\
\hline $\begin{array}{l}\text { Positive } \\
\text { Negative }\end{array}$ & $\begin{array}{l}35 \\
71\end{array}$ & $\begin{array}{c}1.397-4.534 \\
0.021\end{array}$ & & $\begin{array}{l}34 \\
67\end{array}$ & $\begin{array}{c}1.452-4.454 \\
0.001\end{array}$ & \\
\hline Vascular invasion & & 3.550 & & & 2.834 & \\
\hline $\begin{array}{l}\text { Positive } \\
\text { Negative }\end{array}$ & $\begin{array}{l}31 \\
78\end{array}$ & $\begin{array}{c}1.968-6.405 \\
<0.001\end{array}$ & & $\begin{array}{l}30 \\
70\end{array}$ & $\begin{array}{c}1.619-4.959 \\
<0.001\end{array}$ & \\
\hline ASCT2 & & 3.137 & & & 3.183 & \\
\hline $\begin{array}{l}\text { High } \\
\text { Low }\end{array}$ & $\begin{array}{l}33 \\
81\end{array}$ & $\begin{array}{c}1.729-5.690 \\
<0.001\end{array}$ & $\begin{array}{c}2.753(1.222-7.071) \\
0.013\end{array}$ & $\begin{array}{l}30 \\
78\end{array}$ & $\begin{array}{c}1.814-5.585 \\
<0.001\end{array}$ & $\begin{array}{c}2.861(1.324-6.896) \\
0.009\end{array}$ \\
\hline CD98 & & 1.495 & & & 1.333 & \\
\hline $\begin{array}{l}\text { High } \\
\text { Low }\end{array}$ & $\begin{array}{l}47 \\
58\end{array}$ & $\begin{array}{c}0.832-2.686 \\
0.178\end{array}$ & & $\begin{array}{l}44 \\
54\end{array}$ & $\begin{array}{c}0.765-2.325 \\
0.310\end{array}$ & \\
\hline Ki-67 & & 1.887 & & & 1.504 & \\
\hline $\begin{array}{l}\text { High } \\
\text { Low }\end{array}$ & $\begin{array}{l}44 \\
60\end{array}$ & $\begin{array}{c}1.045-3.407 \\
0.035\end{array}$ & & $\begin{array}{l}41 \\
55\end{array}$ & $\begin{array}{c}0.861-2.626 \\
0.151\end{array}$ & \\
\hline CD34 & & 1.379 & & & 1.362 & \\
\hline $\begin{array}{l}\text { High } \\
\text { Low }\end{array}$ & $\begin{array}{l}48 \\
56\end{array}$ & $\begin{array}{c}0.763-2.492 \\
0.287\end{array}$ & & $\begin{array}{l}40 \\
55\end{array}$ & $\begin{array}{c}0.778-2.381 \\
0.279\end{array}$ & \\
\hline $\mathrm{p}-\mathrm{mTOR}$ & & 1.079 & & & 1.168 & \\
\hline $\begin{array}{l}\text { High } \\
\text { Low }\end{array}$ & $\begin{array}{l}50 \\
53\end{array}$ & $\begin{array}{c}0.597-1.948 \\
0.802\end{array}$ & & $\begin{array}{l}46 \\
49\end{array}$ & $\begin{array}{c}0.663-2.506 \\
0.590\end{array}$ & \\
\hline $\begin{array}{l}\text { Abbreviations: } 95 \% \mathrm{Cl}=95 \% \\
\text { non- } \mathrm{AC}=\text { non-adenocarcinoma } \\
\text { Bold entries show statistically s }\end{array}$ & $\begin{array}{l}\text { e interval; } A \\
R=\text { phosph } \\
\text { t difference. }\end{array}$ & $\begin{array}{l}\text { carcinoma; ASC } \\
\text { mammalian tar }\end{array}$ & $\begin{array}{l}\text { SC amino-acid transpo } \\
\text { rapamycin; } p \text {-stage }=p\end{array}$ & $\begin{array}{l}\text { 2; } \mathrm{Cl}=\text { confidence ir } \\
\text { ological stage. }\end{array}$ & & $\mathrm{H}=$ Nishi-Gunma Hospital \\
\hline
\end{tabular}


statistically significant difference in ASCT2 staining was observed between patients with AC (55\%: 36 out of 66$)$ and non-AC (79\%: 30 out of 38$)(P=0.019)$. The median number of CD34-positive vessels was 16 (range, 1-41); thus, 16 was chosen as the cutoff to define a high expression level. The median Ki-67 LI was 17\% (range, 1-82), so $17 \%$ was selected to define high-level expression. High levels of expression of CD34 and Ki-67 LI were detected in $45 \%$ (47 out of 104) and $44 \%$ (46 out of 104) of the tumours, respectively. A total of $39 \%$ (41 out of 104) of the tumours exhibited high-level expression of p-mTOR.

The clinicopathological features of the patients are shown in Table 1. In the NGH cohort, 28 squamous cell carcinomas (SQCs), 6 large cell carcinomas, and 4 NSCLCs were detected in those patients without AC. In the GUH cohort, all non-AC patients presented with SQC, and the positive expression of ASCT2 was significantly higher in SQC compared with AC (70\% vs 40\%, $P<0.001)$. A statistically significant difference in lymphatic permeation, vascular invasion, and ASCT2, CD98, and p-mTOR staining was observed between the NGH and GUH cohorts.

Patient characteristics based on ASCT2 expression. Table 2 shows the characteristics of the tumours in the NGH cohort. In all patients $(n=104)$, positive ASCT2 expression was significantly associated with being male, having an advanced-stage tumour, $\mathrm{T}$ factor, lymph-node metastasis, non-AC, lymphatic permeation, vascular invasion, CD98, Ki-67 LI, and MVD (assessed by CD34 staining). Positive histological staining for ASCT2 in the AC patients was significantly associated with the above variables in addition to p-mTOR, but only with Ki-67 LI in the non-AC patients.

Correlation between ASCT2 expression and different variables. On the basis of Spearman's rank correlation, ASCT2 was significantly correlated with CD98 $(r=0.455, P<0.001)$, Ki-67 $(r=0.413, P<0.001)$, MVD $(r=0.482, P<0.001)$, and $\mathrm{p}-\mathrm{mTOR}$ $(r=0.148, P=0.133)$ in all patients $(n=104)$ from the NGH cohort (Supplementary Table A1). There was also a close correlation with p-mTOR in the AC patients, but not in the non-AC patients. We also validated the correlation between ASCT2 expression and these markers in the GUH cohort $(n=204)$. Consistent with the NGH cohort, ASCT2 expression was positively correlated with CD98 $(r=0.425, P<0.001), \mathrm{Ki}-67 \quad(r=0.475, P<0.001), \mathrm{CD} 34$ $(r=0.496, P<0.001)$, and p-mTOR $(r=0.140, P=0.045)$. Expression of ASCT2 was significantly correlated with CD98, Ki-67, MVD, and mTOR in AC patients $(n=142)$, and with p-mTOR and MVD in non-AC $(n=62)$ subjects.

Patient mortality. In the NGH cohort, the 5-year survival rate and median survival time (MST) for all patients were $51 \%$ and not reached, respectively. The results of univariate and multivariate analyses are shown in Table 3, whereas Figure 2 shows the KaplanMeier survival curve of patients with positive and negative ASCT2 expression. Patient survival was significantly associated with sex, smoking history, disease stage, histology, lymphatic permeation, vascular invasion, ASCT2, and Ki-67 LI, as assessed by a univariate
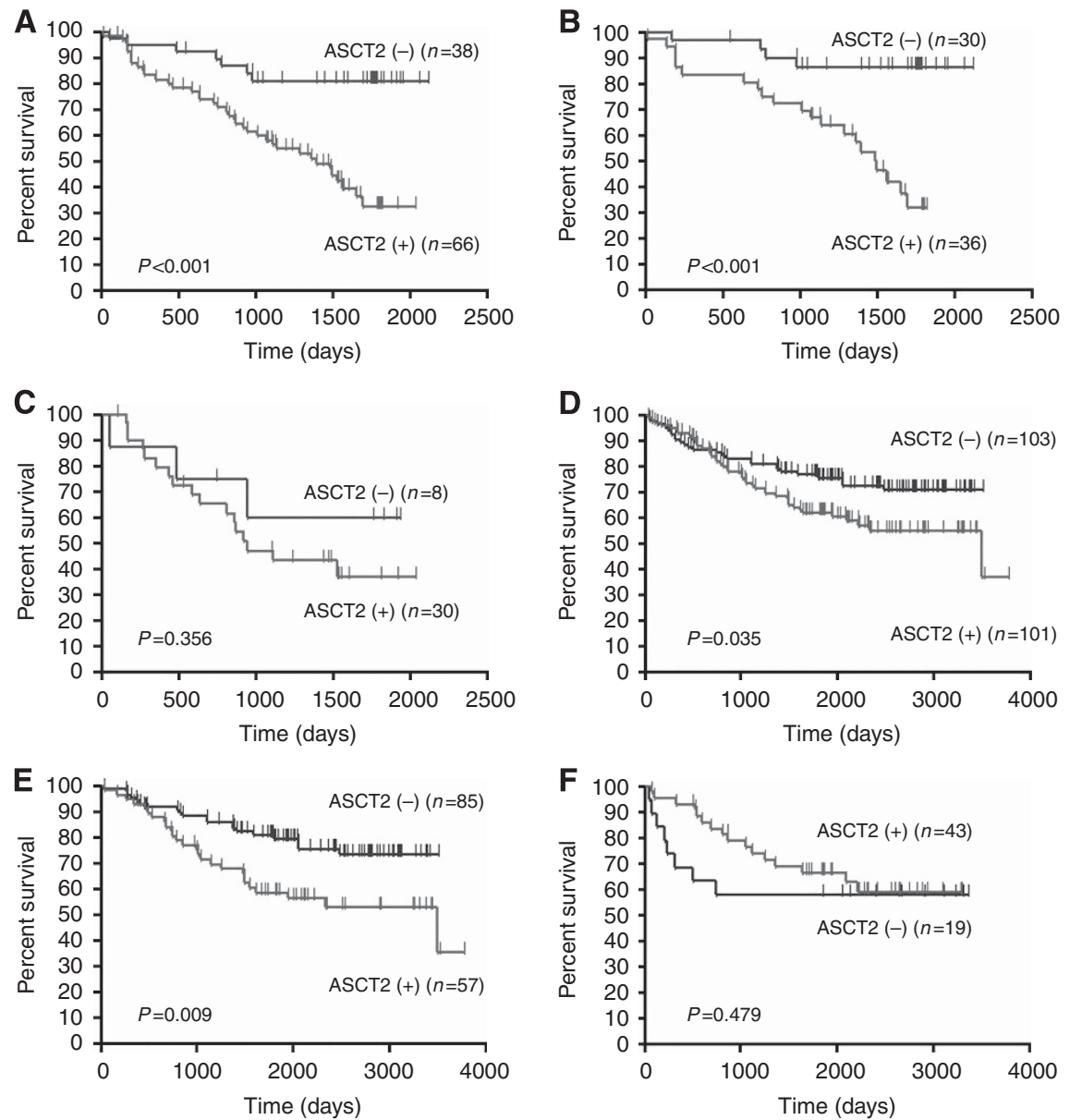

Figure 2. Kaplan-Meier analysis of OS correlated with ASCT2 expression in the NGH and GUH cohorts. A statistically significant difference in OS was observed between the patients with positive and negative tumour expression of ASCT2 in all patients in the NGH (A) and GUH (D) cohorts. When OS was separated by histology, a statistically significant difference was identified in patients with AC in the NGH (B) and GUH (E) cohorts, but not in those with non-AC in NGH (C) and GUH (F). 
analysis. A multivariate analysis confirmed that disease stage and ASCT2 were independent prognostic factors for poor PFS and OS. Expression of ASCT2 was also an independent prognostic indicator for poor outcome in patients with AC.

We next sought to validate the association between ASCT2 expression and survival in the GUH cohort. In the validation cohort (GUH series), the 5-year survival rate and MST for all patients were $69 \%$ and 3491 days, respectively. We compared the OS after surgery between the NGH and GUH cohorts, and found that the OS in the GUH cohort was significantly longer than in the NGH cohort $(P=0.007)$. There was also a (nearly significant) association between positive ASCT2 expression and poor outcome (Figure 2; Table 4). In GUH cohort, we did multivariate analysis using the same prognostic variables in NGH cohort. A multivariate

Table 4. Univariate and multivariate analysis of overall survival and progression-free survival in GUH cohort

Overall survival

\begin{tabular}{|c|c|c|c|c|c|c|}
\hline \multirow[b]{2}{*}{ Variables } & \multicolumn{2}{|c|}{ Univariate analysis } & \multirow{2}{*}{$\begin{array}{c}\begin{array}{c}\text { Multivariate } \\
\text { analysis }\end{array} \\
\text { HR }(95 \% \mathrm{Cl}) \\
P \text {-value }\end{array}$} & \multicolumn{2}{|c|}{ Univariate analysis } & \multirow{2}{*}{$\begin{array}{c}\begin{array}{c}\text { Multivariate } \\
\text { analysis }\end{array} \\
\begin{array}{c}\text { HR }(95 \% \mathrm{Cl}) \\
P \text {-value }\end{array}\end{array}$} \\
\hline & $\begin{array}{l}5 \text {-Year survival } \\
\text { rate }(\%)\end{array}$ & $\begin{array}{c}\text { HR } \\
P \text {-value }\end{array}$ & & $\begin{array}{l}5-\text { Year survival } \\
\text { rate }(\%)\end{array}$ & $\begin{array}{c}\text { HR } \\
P \text {-value }\end{array}$ & \\
\hline Age & & 0.605 & & & 0.743 & \\
\hline $\begin{array}{l}\leqslant 65 \text { years } \\
>65 \text { years }\end{array}$ & $\begin{array}{l}76 \\
66\end{array}$ & $\begin{array}{c}0.359-0.992 \\
0.055\end{array}$ & & $\begin{array}{l}65 \\
57\end{array}$ & $\begin{array}{c}0.473-1.167 \\
0.197\end{array}$ & \\
\hline Sex & & 1.343 & & & 1.128 & \\
\hline $\begin{array}{l}\text { Male } \\
\text { Female }\end{array}$ & $\begin{array}{l}67 \\
76\end{array}$ & $\begin{array}{l}0.835-2.158 \\
0.264\end{array}$ & $\begin{array}{c}0.922(0.633-1.316) \\
0.665\end{array}$ & $\begin{array}{l}59 \\
60\end{array}$ & $\begin{array}{c}0.732-1.737 \\
0.585\end{array}$ & $\begin{array}{c}1.130(0.807-1.553) \\
0.468\end{array}$ \\
\hline Smoking & & 1.606 & & & 1.544 & \\
\hline $\begin{array}{l}\text { Yes } \\
\text { No }\end{array}$ & $\begin{array}{l}65 \\
76\end{array}$ & $\begin{array}{c}0.998-2.584 \\
0.062\end{array}$ & $\begin{array}{c}0.895(0.610-1.328) \\
0.578\end{array}$ & $\begin{array}{l}55 \\
67\end{array}$ & $\begin{array}{c}0.999-2.384 \\
0.051\end{array}$ & $\begin{array}{c}1.242(0.871-1.751) \\
0.227\end{array}$ \\
\hline $\mathrm{p}$-Stage & & 5.981 & & & 13.26 & \\
\hline $\begin{array}{l}\text { I or II } \\
\text { III or IV }\end{array}$ & $\begin{array}{l}78 \\
38\end{array}$ & $\begin{array}{c}3.213-11.13 \\
<0.001\end{array}$ & $\begin{array}{c}3.401(2.059-5.569) \\
<0.001\end{array}$ & $\begin{array}{l}72 \\
16\end{array}$ & $\begin{array}{c}7.147-24.62 \\
<0.001\end{array}$ & $\begin{array}{c}2.175(1.730-2.725) \\
<0.001\end{array}$ \\
\hline Histology & & 1.366 & & & 1.252 & \\
\hline $\begin{array}{l}\text { AC } \\
\text { Non-AC }\end{array}$ & $\begin{array}{l}71 \\
66\end{array}$ & $\begin{array}{c}0.809-2.306 \\
0.323\end{array}$ & $\begin{array}{c}1.029(0.557-1.901) \\
0.926\end{array}$ & $\begin{array}{l}61 \\
57\end{array}$ & $\begin{array}{l}0.780-2.009 \\
0.351\end{array}$ & $\begin{array}{c}0.950(0.721-1.252) \\
0.715\end{array}$ \\
\hline Lymphatic permeation & & 3.232 & & & 3.574 & \\
\hline $\begin{array}{l}\text { Positive } \\
\text { Negative }\end{array}$ & $\begin{array}{l}52 \\
83\end{array}$ & $\begin{array}{c}1.985-5.264 \\
<0.001\end{array}$ & & $\begin{array}{l}39 \\
74\end{array}$ & $\begin{array}{l}2.280-5.602 \\
<0.001\end{array}$ & \\
\hline Vascular invasion & & 3.624 & & & 3.748 & \\
\hline $\begin{array}{l}\text { Positive } \\
\text { Negative }\end{array}$ & $\begin{array}{l}50 \\
80\end{array}$ & $\begin{array}{c}2.170-6.051 \\
<0.001\end{array}$ & & $\begin{array}{l}37 \\
72\end{array}$ & $\begin{array}{c}2.335-6.018 \\
<0.001\end{array}$ & \\
\hline ASCT2 & & 1.657 & & & 1.551 & \\
\hline $\begin{array}{l}\text { High } \\
\text { Low }\end{array}$ & $\begin{array}{l}61 \\
77\end{array}$ & $\begin{array}{c}1.035-2.654 \\
0.035\end{array}$ & $\begin{array}{c}1.179(0.911-1.534) \\
0.209\end{array}$ & $\begin{array}{l}41 \\
54\end{array}$ & $\begin{array}{c}1.008-2.388 \\
0.046\end{array}$ & $\begin{array}{c}1.093(0.868-1.382) \\
0.447\end{array}$ \\
\hline CD98 & & 1.541 & & & 1.710 & \\
\hline $\begin{array}{l}\text { High } \\
\text { Low }\end{array}$ & $\begin{array}{l}63 \\
73\end{array}$ & $\begin{array}{c}0.925-2.569 \\
0.137\end{array}$ & & $\begin{array}{l}48 \\
65\end{array}$ & $\begin{array}{c}1.073-2.724 \\
0.024\end{array}$ & \\
\hline Ki-67 & & 1.748 & & & 1.686 & \\
\hline $\begin{array}{l}\text { High } \\
\text { Low }\end{array}$ & $\begin{array}{l}61 \\
76\end{array}$ & $\begin{array}{c}1.083-2.823 \\
0.031\end{array}$ & & $\begin{array}{l}49 \\
68\end{array}$ & $\begin{array}{c}1.091-2.607 \\
0.018\end{array}$ & \\
\hline CD34 & & 1.876 & & & 1.642 & \\
\hline $\begin{array}{l}\text { High } \\
\text { Low }\end{array}$ & $\begin{array}{l}62 \\
77\end{array}$ & $\begin{array}{c}1.170-3.010 \\
0.012\end{array}$ & & $\begin{array}{l}51 \\
68\end{array}$ & $\begin{array}{c}1.067-2.526 \\
0.024\end{array}$ & \\
\hline p-mTOR & & 1.475 & & & 2.088 & \\
\hline $\begin{array}{l}\text { High } \\
\text { Low }\end{array}$ & $\begin{array}{l}61 \\
73 \\
\end{array}$ & $\begin{array}{c}0.868-2.505 \\
0.130\end{array}$ & & $\begin{array}{l}42 \\
66 \\
\end{array}$ & $\begin{array}{c}1.269-3.437 \\
0.004\end{array}$ & \\
\hline $\begin{array}{l}\text { Abbreviations: } 95 \% \quad \mathrm{Cl}=95 \% \\
\text { non- } \mathrm{AC}=\text { non-adenocarcinoma; } \\
\text { Bold entries show statistically si }\end{array}$ & $\begin{array}{l}\text { onfidence interval; } \\
\text { mTOR = phosphorylat } \\
\text { ficant difference. }\end{array}$ & $\begin{array}{l}\text { adenocarcinom } \\
\text { mammalian tar }\end{array}$ & $\begin{array}{l}\mathrm{CT} 2=\mathrm{ASC} \text { amino-acid } \\
\text { apamycin; } \text { p-stage }=\text { patt }\end{array}$ & $\begin{array}{l}\text { transporter 2; GUH } \\
\text { ological stage. }\end{array}$ & unma University & spital; $\quad H R=$ hazard ratio; \\
\hline
\end{tabular}


analysis indicated that disease stage was an independent prognostic factor for poor outcome in all patients with NSCLC (Table 4). Survival was then examined in relation to histological sub-type. In patients with $\mathrm{AC}$, tumour stage and ASCT2 expression were independent predictors of poor OS in a multivariate analysis (Table 5). In contrast, ASCT2 expression was not associated with poor prognosis in patients with SQC.

\section{DISCUSSION}

This is the first report to evaluate the prognostic significance of ASCT2 expression in patients with surgically resected NSCLC. Our data clearly demonstrate that ASCT2 expression was an independent prognostic marker for poor outcome after surgery in patients

\section{Table 5. Univariate and multivariate analysis of OS and PFS in AC patients (GUH cohort)}

Overall survival

\begin{tabular}{|c|c|c|c|c|c|}
\hline \multicolumn{2}{|c|}{ Univariate analysis } & $\begin{array}{c}\text { Multivariate } \\
\text { analysis }\end{array}$ & \multicolumn{2}{|c|}{ Univariate analysis } & Multivariate analysis \\
\hline $\begin{array}{c}\text { 5-Year survival } \\
\text { rate (\%) }\end{array}$ & $P$-value & $\begin{array}{c}\text { HR }(95 \% \mathrm{Cl}) \\
P \text {-value }\end{array}$ & $\begin{array}{c}\text { 5-Year survival } \\
\text { rate (\%) }\end{array}$ & $P$-value & $\begin{array}{c}\mathrm{HR}(95 \% \mathrm{Cl}) \\
P \text {-value }\end{array}$ \\
\hline
\end{tabular}

\section{Age}

$\leqslant 65$ years

$>65$ years

\section{Sex}

Male

Female

67

78

67

0.075

68

56

\subsection{5}

0.267

\begin{tabular}{c|c}
$0.891(0.592-1.324)$ & 58 \\
0.665 & 63
\end{tabular}

63

0.509

$1.056(0.732-1.506)$

74

0.046

$0.898(0.603-1.354)$

52

68

0.038

$1.196(0.823-1.719)$

77

0.578

0.343

\section{p-Stage}

\begin{tabular}{|l|l}
\hline I or II & 82 \\
\hline III or IV
\end{tabular}

III or IV

\begin{tabular}{l|l}
82 & $<0.001$
\end{tabular}

$2.186(1.614-2.967)$

$<0.001$

75
15

$<0.001$

2.175 (1.730-2.725)

$<0.001$

\section{Lymphatic permeation}

\begin{tabular}{|l|l|l|l|l|l|}
\hline Positive & 46 & $<0.001$ & & 32 & 78 \\
Negative & 86 & & 0.001 \\
\hline
\end{tabular}

\section{Vascular invasion}

\begin{tabular}{|c|c|c|c|c|c|c|}
\hline $\begin{array}{l}\text { Positive } \\
\text { Negative }\end{array}$ & $\begin{array}{l}44 \\
84\end{array}$ & $<0.001$ & & $\begin{array}{l}27 \\
77\end{array}$ & $<0.001$ & \\
\hline \multicolumn{7}{|l|}{ ASCT2 } \\
\hline $\begin{array}{l}\text { High } \\
\text { Low }\end{array}$ & $\begin{array}{l}59 \\
79\end{array}$ & 0.009 & $\begin{array}{c}1.424(1.057-1.929) \\
0.012\end{array}$ & $\begin{array}{l}48 \\
69\end{array}$ & 0.024 & $\begin{array}{c}1.205(0.919-1.583) \\
0.177\end{array}$ \\
\hline
\end{tabular}

\section{CD98}

\begin{tabular}{l|l}
\hline High & \\
\hline
\end{tabular}

Low

61
73

0.270

37

66

0.021

\section{Ki-67}

\begin{tabular}{l|l}
\hline High & \\
\hline
\end{tabular}

\section{CD34}

\section{High}

Low

0.001

51
78

0.001

0.006

58
77

p-mTOR

High

Low

64

75

0.243

Abbreviations: $95 \% \mathrm{Cl}=95 \%$ confidence interval; $\mathrm{AC}=$ adenocarcinoma; $\mathrm{ASCT} 2=\mathrm{ASC}$ amino-acid transporter 2; GUH=Gunma University Hospital; HR = hazard ratio; OS = overall survival; $\mathrm{PFS}=$ progression-free survival; $\mathrm{p}-\mathrm{mTOR}=$ phosphorylation of mammalian target of rapamycin; $\mathrm{p}$-stage = pathological stage.

Bold entries show statistically significant difference. 
with NSCLC, particularly AC. Although the expression of ASCT2 was increased significantly in non-AC patients compared with AC patients, ASCT2 in AC patients was more closely associated with disease stage, lymphatic permeation, vascular invasion, CD98, cell proliferation, angiogenesis, and mTOR phosphorylation. Moreover, two independent cohorts demonstrated that ASCT2 was an independent predictor of poor outcome in AC patients. Our validated data suggest that ASCT2 has an important role in the aggressiveness and metastasis of lung cancer, particularly AC.

Only two previous studies reported enhanced expression of ASCT2 in primary human colorectal AC and prostate cancer, suggesting a close relationship between its expression and poor prognosis (Whitte et al, 2002; Li et al, 2003). Therefore, further study is warranted to investigate the clinical significance of ASCT2 expression in other human cancers. Our study focussed on the clinicopathological significance of ASCT2 expression in patients with lung cancer. Importantly, our study included validating data from an independent cohort, and evaluated the expression and activation of the mTOR signalling pathway, which is related to protein synthesis. Previously, we demonstrated that LAT1 is required for the upregulation of $\mathrm{mTOR}$ in lung cancer, which was supported by in vitro and in vivo data (Imai et al, 2010; Kaira et al, 2011). Fuchs et al (2007) reported that LAT1 provides essential amino acids for tumour cell growth via mTOR-stimulated translation, and that ASCT2 maintains the cytoplasmic aminoacid pool necessary to promote LAT1 function. Therefore, they demonstrated that both LAT1 and ASCT2 are highly expressed in human cancers, and that there is reciprocal regulation among LAT1, ASCT2, and mTOR. Recent studies demonstrated that the inhibition of amino-acid transporters reduces the p-mTOR, p70 ribosomal S6 kinase, and 4E-binding protein-1. This leads to the induction of apoptosis by depleting the intracellular amino acids required for cancer growth, and induces a cell-cycle arrest at G1 phase (Liu et al, 2004; Yamauchi et al, 2009; Imai et al, 2010; Kim et al, 2010). Because the p-mTOR is closely related to the survival and metastasis of cancer cells, the inhibition of amino-acid transporters such as LAT1 or ASCT2 may suppress tumour growth by decreasing mTOR phosphorylation. However, additional studies are needed to investigate the mechanism by which the inhibition of ASCT2 expression inhibits tumour growth.

We found that ASCT2 could be a pathological marker for predicting poor outcome after surgery, and that it was closely associated with tumour cell proliferation and angiogenesis in patients with AC, but not in non-AC patients (predominantly SQC). However, the reasons for the differential effects and levels of ASCT2 protein expression between $\mathrm{AC}$ and non-AC patients remain unclear. Expression of LAT1 is significantly higher in patients with SQC than in those with AC (Kaira et al, 2008). The expression of ASCT2 analysed by histological sub-type is similar to that of LAT1 (Kaira et al, 2008). In our study, ASCT2 seemed to have an important role in tumour cell proliferation and angiogenesis in AC patients, suggesting a close relationship between ASCT2 expression and prognosis. However, little is known about the clinical significance of the expression pattern of ASCT2 in human tumour tissues. Therefore, it is necessary to investigate ASCT2 expression in various types of cancer using human cancer specimens. Presently, clinicopathological studies of ASCT2 expression are ongoing in gastrointestinal cancer, hepatobiliary cancer, multiple myeloma, ovarian tumours, and breast cancer.

There are several limitations to our study. First, the number of non-AC patients included was small, and the histological distribution of non-AC disease was different between the NGH and GUH cohorts, which may have biased our results. The frequency of SQC patients was significantly higher in the GUH cohort $(100 \%, 62$ out of 62$)$ than in the NGH cohort $(74 \%, 28$ out of 38$)(P<0.01)$. However, a survival analysis of the non-AC patients seemed to give comparable results in the two cohorts.
Second, the frequency of ASCT2 expression in the GUH cohort was significantly lower than in the NGH cohort. Therefore, tumour aggressiveness and prognosis after surgery may be different between these cohorts. In addition, there was a significant difference in lymphatic permeation, vascular invasion, and biomarker expression (CD98 and p-mTOR) between the NGH and GUH cohorts. Although we cannot describe the detailed reason for these differences, the tumour characteristics may be more aggressive in $\mathrm{NGH}$ than in $\mathrm{GUH}$, considering that the expression of ASCT2 has a significant relationship with lymphatic permeation, vascular invasion, CD98, and p-mTOR. The present study showed that the expression of ASCT2 was closely associated with lymphatic permeation, vascular invasion, and cell proliferation (Ki-67). Therefore, these factors were excluded from the multivariate analysis to assess ASCT2 as an independent prognostic factor and also to resolve confounding issue. Finally, median survival was not reached for the NGH cohort. In this cohort, five patients were lost to follow-up. The NGH cohort may have a potential for selection bias, because of the issues with loss to follow-up in this cohort. Moreover, the sample size was markedly different between NGH and GUH cohort. These findings may be possible reasons for this discrepancy for survival analysis.

In conclusion, the expression of ASCT2 is a validated predictive marker for poor prognosis in patients with $\mathrm{AC}$, and is significantly correlated with tumour aggressiveness, cell proliferation, angiogenesis, and mTOR phosphorylation. The inhibition of ASCT2 could be a future therapeutic strategy for lung cancer. However, additional studies are needed to assess the biological significance of inhibiting ASCT2 in human cancer cells.

\section{ACKNOWLEDGEMENTS}

This study was supported by a grant from the Advanced Research for Medical Products Mining Program of the National Institute of Biomedical Innovation.

\section{CONFLICT OF INTEREST}

The authors declare no conflict of interest.

\section{REFERENCES}

Altman A, Cardenas JM, Houghten RA, Dixon FJ, Theofilopoulos AN (1984) Antibodies of predetermined specificity against chemically synthesized peptides of human interleukin 2. Proc Natl Acad Sci 81: 2176-2180.

Brundage MD, Davies D, Mackillop WJ (2002) Prognostic factors in non-small cell lung cancer: a decade of prognosis. Chest 122: 1037-1057.

Chairoungdua A, Kanai Y, Matsuo H, Inatomi J, Kim DK, Endou H (2001) Identification and characterization of a novel member of the heterodimeric amino acid transporter family presumed to be associated with an unknown heavy chain. J Biol Chem 276: 49390-49399.

Fuchs BC, Bode BP (2006) Amino acid transporters ASCT2 and LAT1 in cancer: Partners in crime? Semin Cancer Biol 15: 254-266.

Fuchs BC, Finger RE, Onan MC, Bode BP (2007) ASCT2 silencing regulates mammalian target of rapamycin growth and survival signaling in human hepatoma cells. Am J Physiol Cell Physiol 293: C55-C63.

Furuya M, Horiguchi J, Nakajima H, Kanai Y, Oyama T (2012) Correlation of L-type amino acid transporter 1 and CD98 expression with triple negative breast cancer prognosis. Cancer Sci 103: 382-389.

Ichinoe M, Mikami T, Yoshida T, Igawa I, Tsuruta T, Nakada N, Anzai N, Suzuki Y, Endou H, Okayasu I (2011) High expression of L-type aminoacid transporter 1 (LAT1) in gastric carcinomas: Comparison with noncancerous lesions. Pathol Int 61: 281-289. 
Imai $\mathrm{H}$, Kaira K, Oriuchi N, Shimizu K, Tominaga $\mathrm{H}$, Yanagitani N, Sunaga N, Ishizuka T, Nagamori S, Promchan K, Nakajima T, Yamamoto N, Mori M, Kanai Y (2010) Inhibition of L-type amino acid transporter 1 has antitumor activity in non-small cell lung cancer. Anticancer Res 30: 4819-4828.

Kaira K, Oriuchi N, Imai H, Shimizu K, Yanagitani N, Sunaga N, Hisada T, Tanaka S, Ishizuka T, Kanai Y, Endou H, Nakajima T, Mori M (2008) Prognostic significance of L-type amino acid transporter 1 expression in resectable stage I-III nonsmall cell lung cancer. Br J Cancer 98: 742-748.

Kaira K, Oriuchi N, Takahashi T, Nakagawa K, Ohde Y, Okumura T, Murakami H, Shukuya T, Kenmotsu H, Naito T, Kanai Y, Endo M, Kondo H, Nakajima T, Yamamoto N (2011) LAT1 expression is closely associated with hypoxic markers and mTOR in resected non-small cell lung cancer. Am J Transl Res 3: 468-478.

Kaira K, Sunose Y, Arakawa K, Ogawa T, Sunaga N, Shimizu K, Tominaga H, Oriuchi N, Itoh H, Nagamori S, Kanai Y, Segawa A, Furuya M, Mori M, Oyama T, Takeyoshi I (2012) Prognostic significance of L-type amino acid transporter 1 expression in surgically resected pancreatic cancer. Br J Cancer 107: 632-638.

Kanai Y, Segawa H, Miyamoto K, Uchino H, Takeda E, Endou H (1998) Expression cloning and characterization of a transporter for large neutral amino acids activated by the heavy chain of 4F2 antigen (CD98). J Biol Chem 273: 23629-23632.

Kawaguchi T, Takada M, Kubo A, Matsumura A, Fukai S, Tamura A, Saito R, Kawahara M, Maruyama Y (2010) Gender, histology, and time of diagnosis are important factors for prognosis: analysis of 1499 never-smokers with advanced non-small cell lung cancer in Japan. J Thorac Oncol 5: 1011-1017.

Kekuda R, Prasad PD, Fei YJ, Torres-Zamorano V, Sinha S, Yang-Feng TL, Leibach FH, Ganapathy V (1996) Cloning of the sodium-dependent, broad-scope, neutral amino acid transporter Bo from a human placental choriocarcinoma cell line. J Biol Chem 271: 18657-18661.

Khunweeraphong N, Nagamori S, Wiriyasermkul P, Nishinaka Y, Wongthai P, Ohgaki R, Tanaka H, Tominaga H, Sakurai H, Kanai Y (2012) Establishment of stable cell lines with high expression of heterodimers of human $4 \mathrm{~F} 2 \mathrm{hc}$ and human amino acid transporter LAT1 or LAT2 and delineation of their differential interaction with $\alpha$-alkyl moieties. J Pharmacol Sci 119: 368-380.

Kim CS, Moon IS, Park JH, Shin WC, Chun HS, Lee SY, Kook JK, Kim HJ, Park JC, Endou H, Kanai Y, Lee BK, Kim do K (2010) Inhibition of L-type amino acid transporter modulates the expression of cell cycle regulatory factors in KB oral cancer cells. Biol Pharm Bull 33: 1117-1121.

Kogure Y, Ando M, Saka H, Chiba Y, Yamamoto N, Asami K, Hirashima T, Seto T, Nagase S, Otsuka K, Yanagihara K, Takeda K, Okamoto I, Aoki T, Takayama K, Yamasaki M, Kudoh S, Katakami N, Miyazaki M, Nakagawa K (2013) Histology and smoking status predict survival of patients with advanced non-small-cell lung cancer. Results of West Japan Oncology Group (WJOG) Study 3906L. J Thorac Oncol 8: 753-758.

Li R, Younes M, Frolov A, Wheeler TM, Scardino P, Ohori M, Ayala G (2003) Expression of neutral amino acid transporter ASCT2 in human prostate. Anticancer Res 23: 3413-3418.

Liu XM, Reyna SV, Ensenat D, Peyton KJ, Wang H, Schafer AI, Durante W (2004) Platelet-derived growth factor stimulates LAT1 gene expression in vascular smooth muscle: role in cell growth. FASEB $J$ 18: $768-770$.

Mountain CF (1997) Revision in the international system for staging lung cancer. Chest 11: 1710-1717.

Nakamura H, Ando K, Shinmyo T, Morita K, Mochizuki A, Kurimoto N, Tatsunami S (2011) Female gender is an independent prognostic factor in non-small-cell lung cancer: a meta-analysis. Ann Thorac Cardiovasc Surg 17: $469-480$.

Nakanishi K, Ogata S, Matsuo H, Kanai Y, Endou H, Hiroi S, Tominaga S, Aida S, Kasamatsu H, Kawai T (2007) Expression of LAT1 predicts risk of progression of transitional cell carcinoma of the upper urinary tract. Virchows Arch 451: 681-690.

Nawashiro H, Otani N, Shinomiya N, Fukui S, Ooigawa H, Shima K, Matsuo H, Kanai Y, Endou H (2006) L-type amino acid transporter 1 as a potential molecular target in human astrocytic tumors. Int J Cancer 119: $484-492$.

Sakata T, Ferdous G, Tsuruta T, Satoh T, Baba S, Muto T, Ueno A, Kanai Y, Endou H, Okayasu I (2009) L-type amino acid transporter 1 as a novel biomarker for high-grade malignancy in prostate cancer. Pathol Int 59: $7-18$.

Whitte D, Ali N, Carlson N, Younes M (2002) Overexpression of the neutral amino acid transporter ASCT2 in human colorectal adenocarcinoma. Anticancer Res 22: 2555-2557.

Yamauchi K, Sakurai H, Kimura T, Wiriyasermkul P, Nagamori S, Kanai Y, Kohno N (2009) System L amino acid transporter inhibitor enhances anti-tumor activity of cisplatin in a head and neck squamous cell carcinoma cell line. Cancer Lett 276: 95-101.

Yanagida O, Kanai Y, Chairoungdua A, Kim DK, Segawa H, Nii T, Cha SH, Matsuo H, Fukushima J, Fukasawa Y, Tani Y, Taketani Y, Uchino H, Kim JY, Inatomi J, Okayasu I, Miyamoto K, Takeda E, Goya T, Endou H (2001) Human L-type amino acid transporter 1 (LAT1): characterization of function and expression in tumor cell lines. Biochim Biophys Acta 1514: 291-302.

This work is published under the standard license to publish agreement. After 12 months the work will become freely available and the license terms will switch to a Creative Commons AttributionNonCommercial-Share Alike 3.0 Unported License.

Supplementary Information accompanies this paper on British Journal of Cancer website (http://www.nature.com/bjc) 